\title{
What We Shall Know Only Tomorrow
}

\author{
Peter Hagoort \\ Max Planck Institute for Psycholinguistics, Nijmegen, The Netherlands
}

In contrast to a widely held belief among politicians and science managers, the course of science is unpredictable and difficult to steer. The reason is that management is based on the knowledge of today, whereas science is about the knowledge of tomorrow. As Sir Karl Popper (1957) remarked: "We cannot anticipate today what we shall know only tomorrow." Anyone who specifies the key issues in the field of brain and language for the first hundred years of the new millennium therefore runs the risk of becoming a fool in the light of history's hindsight and of the knowledge and discoveries still hidden behind the curtains of the third millennium. This being said, for the sake of providing the third millennium historians of science with materials for reconstructing the belief systems of the old millennium scientists, one should be willing to play the role of jester in science's hall of fame. No doubt this third millennium issue will contain more off-track predictions than spot-on foresight. Future generations will decide where my remarks fell on the line between stupidity and wisdom. To protect myself against an overdose of speculation I will discuss key issues in the context of today's science of brain and language.

One of the key issues will be the development of a more intricate science of brain and language. The classical neuropsychological approach has exploited the functional fractionation of cognitive systems as a result of brain lesions. No doubt this has resulted in a great number of relevant findings with important theoretical implications. However, this approach has also too often ignored the issue of how neuropsychological accounts fare in the light of knowledge about the principles of neural organization. A very recent example in case is Grodzinsky's theory about the neurology of syntax, and

I thank Colin Brown and Peter Indefrey for their helpful comments.

Address correspondence and reprint requests to Peter Hagoort, F. C. Donders Centre for Cognitive Neuroimaging and Max Planck Institute for Psycholinguistics, P.O. Box 310, NL6500 AH Nijmegen, The Netherlands. E-mail: p.hagoort@mpi.nl. 
more in particular the role of Broca's area (Grodzinsky, in press). Grodzinsky gives a very specific and detailed account of the role of Broca's area and adjacent cortex in the context of linguistic theory. In this account Broca's area is claimed to specifically handle intra-sentential dependency relations. The body of evidence for this proposal is the error patterns of neurological patients with Broca's aphasia. However, in sharp contrast to the specifics of the linguistic proposal is the complete underspecification of the neural underpinnings of this claim. For instance, recent neuroanatomical analyses (Uylings et al., 1999) have shown that it is not so easy to delineate Broca's area on the basis of landmark sulcal patterns, and that, moreover, there is a lot of individual variation in the exact size and shape of Broca's area. Grodzinsky does not address any of these issues. A detailed specification of the lesions in the patients on which he bases his claims is lacking. A careful lesion overlap analysis (cf. Dronkers, 1996) is not presented. About the neural architecture of syntax he concludes that "syntax, though less localized than previously believed, is localized in the left hemisphere, and is distinct from other, seemingly related, intellectual capacities"'(Grodzinsky, in press). That syntax is localized in the left hemisphere will certainly not come as a big surprise to neurolinguists and neuroscientists. What we are in need of, however, are answers to the following types of questions: What are the neuroanatomical characteristics that would make Broca's area suitable for handling intra-sentential dependency relations? Is Broca's area only involved in certain syntactic operations or is it also part of networks of brain areas performing other cognitive operations? According to Grodzinsky, Broca's area only handles intra-sentential dependency relations. However, there is solid evidence from brain imaging studies that Broca's area and adjacent cortex is also involved in, for instance, the phonological analysis (e.g., converting orthographic letter strings into phonological output codes) of single words (cf. Hagoort et al., 1999). This relates to the issue whether from the perspective of neural organization it makes sense to conceptualize domain specificity in terms of cytoarchitectonic areas that are exclusively involved in one and only one function. Another relevant question that is untouched in Grodzinsky's account: How is the computation of intra-sentential dependency relations in Broca's area hooked up to the activity of other areas subserving different aspects of syntax? Very interesting suggestions from a neural organization perspective for answers to these kinds of questions can be found in Mesulam (1998). I used this example not to belittle Grodzinsky's account or to deny that it has valuable aspects. I took it solely as a representative example of a long-standing approach in neurolinguistics. In the context of the third millennium this example highlights an issue that will become more pressing in the near future. That is, that we not only need well-articulated models of language functions, but also well-articulated models of the brain to allow the integration of our understanding of language and our understanding of the brain into a true cognitive neuroscience of language. The classical 
neurolinguistic approach has not taken the brain into consideration in a serious enough manner. In the modern era of brain imaging and the growth of the neurosciences this will in my eyes become one of the key issues.

Strangely enough, often connectionist style models of language processing have also not taken brain data seriously enough. For instance, in most connectionist models of sentence processing the by now substantial body of electrophysiological data on sentence processing are not considered as important neural constraints on the modelling approach (cf. Hagoort, Brown, \& Osterhout, 1999). The fact that qualitatively different ERP-effects are observed to semantic binding operations and parsing, respectively, strongly suggests that the neural basis of these two types of sentence processes is to an interesting degree nonoverlapping. The ERP data favor a view in which semantic and syntactic processes have processing and/or representational uniqueness relative to each other. This does not necessarily mean that semantic integration and parsing are informationally encapsulated (modular), but it does imply domain specificity for semantic and syntactic processing operations. The neural basis of syntactic computations can therefore not be collapsed into a general-purpose language processor that operates only on the cooccurrence frequencies of the word input, or in which semantic and syntactic information types do not result in clearly different states of the processing/representational landscape (cf. Tabor et al., 1997). Neurologically plausible constraint-based models of sentence processing should be able to account for the ERP findings. However, in most cases this type of brain data is not addressed at all in the connectionist style modeling of language. Clearly with the advancement of the cognitive neuroimaging tools, our knowledge about the neural architecture of language will increase. Connectionist accounts of language must face the increasing body of brain data upfront, or otherwise they will become obsolete before the first decennium of the third millennium is over.

A final key issue concerns the exploitation of neural plasticity for the recovery of aphasia and other language disorders. Neural plasticity in the adult brain seems more substantial than for a long time was thought to be the case according to the standard view on the mature brain. If this is so, the question is on the table how to target neural plasticity effectively for functional recovery. I do not think that as of yet we have a very good handle on how neural plasticity and functional recovery, or the potential for functional recovery, are related. Nevertheless, it will be a challenge in the third millennium to investigate this issue and to optimize treatment procedures for attaining maximal functional recovery within the limits that neural plasticity sets.

\section{REFERENCES}

Dronkers, N. F. 1996. A new region for coordinating speech articulation. Nature, 384, 159161. 
Grodzinsky, Y. (in press). The neurology of syntax: Language use without Broca's area. Behavioral and Brain Sciences, 23.

Hagoort, P., Brown, C. M., \& Osterhout, L. 1999. The neurocognition of syntactic processing. In C. M. Brown \& P. Hagoort (Eds.), The neurocognition of language (pp. 273-316). Oxford, UK: Oxford University Press.

Hagoort, P., Indefrey, P., Brown, C, Herzog, H., Steinmetz, H., \& Seitz, R. 1999. The neural circuitry involved in the reading of German words and pseudowords: A PET study. Journal of Cognitive Neuroscience, 11, 383-398.

Mesulam, M.-M. 1998. From sensation to cognition. Brain, 121, 1013-1052.

Popper, K. 1957. The poverty of historicism. London: Routledge \& Kegan Paul.

Tabor, W, Juliano, C., \& Tanenhaus, M. K. 1997. Parsing in a dynamical system: An attractorbased account of the interaction of lexical and structural constraints in sentence processing. Language and Cognitive Processes, 12, 211-271.

Uylings, H. B. M., Malofeeva, L. I., Bogolepova, I. N., Amunts, K., \& Zilles, K. 1999. Broca’s language area from a neuroanatomical and developmental perspective. In C. M. Brown \& P. Hagoort (Eds.), The neurocognition of language (pp. 273-316). Oxford, UK: Oxford University Press. 